\title{
PROTECTIVE EFFECT OF SOYBEAN OIL ON MICE LUNG TISSUE INJURY AND ALVEOLAR HEMORRHAGE INDUCED BY BISPHENOL A
}

\author{
Sadia Shaukat, Hina Kundi*, Qazi Waheed Ullah, Nazish Waheed, Farah Deeba**, Shazia Iftikhar*** \\ Rehman Medical College, Peshawar Pakistan, *Fazaia Medical College, Islamabad Pakistan, **Pak Institute Medical College, Peshawar Pakistan, \\ ***Khyber Medical College, Peshawar Pakistan
}

\begin{abstract}
Objective: To evaluate histomorphological effects of Soybean oil supplementation on lung tissue injury and alveolar hemorrhage induced by Bisphenol A (BPA).

Study Design: Laboratory-based experimental study.

Place and Duration of Study: Anatomy department, Army Medical College, Rawalpindi and National Institute of Health, Islamabad, from Nov 2015 to Nov 2016.

Methodology: Forty (40) healthy BALB/c mice of 9-11 weeks of age, weighing between 30-37gm were housed in a controlled environment at National Institute of Health. Group 1 (10) was control group. Group 2 (10) was given a daily dose of 50 milligram/kilogram body weight of Bisphenol A and group 3 (10) was given a daily dose of 500 milligram of Soybean oil and group 4 (10) was concurrently given Bisphenol A and Soybean oil with daily doses of 50 milligram/kilogram body weight and 500 milligrams. After a period of 8 weeks, animals were dissected 24 hours after receiving the last dosage. Lung wet weight, animal weight and relative body tissue weight index (RTBWI) were calculated. Tissue processing \& staining was done. Alveolar hemorrhage was histomorphologically and statistically analysed using SPSS-21.

Results: On microscopic examination, alveolar hemorrhage (AH) was observed in $10(100 \%)$ group 2 specimens with increase in RTBWI and whereas only $5(50 \%)$ of group 4 specimens had alveolar hemorrhage with slight improvement in relative body tissue weight index (RTBWI).

Conclusion: Bisphenol A (BPA) induced lung injury as evident by intraalveolar hemorrhage, blood vessel congestion and increased RTBWI ratio were ameliorated by concomitant administration of Soybean oil.
\end{abstract}

Keywords: Alveolar hemorrhage, Bisphenol a (BPA), Relative body tissue weight index (RTBWI), Soybean oil.

This is an Open Access article distributed under the terms of the Creative Commons Attribution License (https://creativecommons.org/licenses/by-nc/4.0/), which permits unrestricted use, distribution, and reproduction in any medium, provided the original work is properly cited.

\section{INTRODUCTION}

Bisphenol A (BPA), a synthetically produced chemical is one of the chief constituent of polycarbonate plastics, detectable in commonly used household products, food packing, tin linings, dental sealants and thermally generated receipts. Substantial levels of BPA have been quantified in air, drinking water, rural areas encompassing and even in the dust ${ }^{1}$. Independently, high and low-dosage exposures to BPA associates structural and functional impairment in prostate, testis, mammary gland, uterus, cardiovascular system, along with alterations in brain structure, chemistry and behaviours in experimental animals. BPA, disrupts cytokine signalling regulation responsible for a variety of diseases, comprising allergy, lung inflammation, autoimmune dysfunction, and even tumours ${ }^{2}$. Chronic exposures to low dosage of BPA disrupts lung microarchitecture exhibiting collapsed alveoli, intra-alveolar haemorrhages, congested blood vessels, collagen fibre deposition, and inflammatory cellular infiltration around bronchi or blood vessels. A substantial proliferation of

Correspondence: Dr Sadia Shaukat, Assistant Professor of Anatomy, Rehman Medical College, Peshawar Pakistan

Received: 22 Oct 2020; revised received: 01 Mar 2021; accepted: 04 Mar 2021 alveolar macrophages and inducible nitric oxide synthase (i-NOS) immunoreaction are also documented ${ }^{3}$. Changes observed in mild dose BPA treated groups in another study, propose increase in immunoreaction for i-NOS and release of reactive oxygen species (ROS). One of the metabolite of BPA, a quinine radical leads to formation of ROS. In addition, BPA increases vascular permeability and causes direct injury to vascular endothelium. Oxidative stress also causes secretion of Cyclophilin A from vascular smooth muscles, stimulating muscle proliferation. These changes form the basis of vascular congestion and extravasation of RBCs within the alveolar lumen, observed as haemorrhagic foci on the surface of the lungs. Chronic exposure to BPA causes enhanced concentrations of MDA (malondialdehyde), reduction in SOD (superoxide dismutase) concentrations, and up regulates Interleukin-18 (IL-18) demonstration in animal lung tissue. In addition, augmented expression of follistatin-like1 (FSTL1) and moderated disintegrin and metalloproteinase with thrombospondin motif 5 (ADAMTS5) expression is documented.

Chronic inflammatory condition associated with BPA lead to dysregulation in the levels of chemokines, 
cytokines and various growth factors disrupting recruitment of cells. In addition, excessive pro-fibrotic IL13 and/or TGF $\beta 1$ production disrupts healing process and culminates into a pathogenic fibrotic response ${ }^{5}$, eventually leading to endothelial cell adhesion and injury and parenchymal migration of neutrophils from the vascular space. This accumulation releases oxidants and proteases that directly injure vascular endothelial cells. This causes inflammatory cellular infiltration, vascular congestion, and extravasated RBCs of the lung tissue. Chronic BPA exposure especially to its metabolite 4-Methyl-2, 4-bis 4-hydroxyphenyl pent-1ene alters lung functional capacity and type 2 alveolar cell growth, leading to pulmonary inflammatory diseases and lung fibrosis ${ }^{6}$. BPA modifies development, maturation and functioning of lungs during prenatal and postnatal life, leading to asthma and allergic lung inflammation especially during childhood ${ }^{7}$.

Soybeans consist of bioactive plant compounds including phytic acid, saponins and isoflavones. Isoflavones with antioxidant polyphenols additionally known as phytoestrogens, contain weak estrogenic \& antioxidant activity ${ }^{8}$. Phytic acid present in Soybean oil is likewise documented to have antioxidant and chelating properties. Saponins have cholesterol lowering properties ${ }^{9}$. Furthermore, molybdenum, phosphorus vitamin K1, folate, manganese, copper, and thiamine also have significant effects.

This study was objective to evaluate acute lung injury and alveolar haemorrhage induced by BPA and antioxidant protection by dietary supplementation with soybean oil.

\section{METHODOLOGY}

Laboratory-based experimental study was conducted in department of Anatomy, Army Medical College, Rawalpindi with cooperation of National Institute of Health and Pathology Department, from November 2015 to November 2016, after ethics approval of authorities of Army Medical College (ERC/SA-16/Dr. Sadia Shaukat). Sigma Aldrich provided the experimental chemical Bisphenol A.

Forty healthy adult BALB/c lab mice, 9-11 weeks old and weighing 30-40 gm were selected randomly for the experiment. Mice were lodged in a well-ventilated, clean room of $\mathrm{NIH}$ at room temperature maintained between $20-26^{\circ} \mathrm{C}$ and were allowed 12 hours of darklight sleep cycle. Mice were contained in polystyrene cages and fed with specifically designed Soybean restricted lab diet (to eliminate phytoestrogen component of pallets) to diminish circumstantial BPA exposure.
Water was available ad libitum in polystyrene bottles. Complete doses were administered through oral gavage daily for a total period of eight weeks.

Mice in group 1 were control animals. Experimental group 2 was administered with $50 \mathrm{mg} / \mathrm{kg} /$ day of BPA. Group 3 administered with $500 \mathrm{mg} /$ day of Soybean oil and mice in experimental group 4 were administered $50 \mathrm{mg} / \mathrm{kg} /$ day of BPA and $500 \mathrm{mg} /$ day of Soybean oil simultaneously. The body weight of all the animals were recorded at the start of the study as well as before the sacrifice of animals. At the end of 8week experiment, animals were euthanized, sacrificed, $10 \%$ Formalin was injected into trachea between the cartilaginous rings until the lungs were inflated with almost $2-3 \mathrm{ml}$ of fluid, fresh lung specimens were dissected out and weighed. The weight was recorded in gm with the help of a digital analytical balance. The RTBWI was calculated by using the formula ${ }^{10}$. RTBWI= weight of organ in gm X 100/weight of body in gm.

Changes such as presence or absence of haemorrhagic foci on lung surface were observed and recorded Lung tissue processing was done in $10 \%$ formalin and 5 -micron thick sections were cut using rotary microtome. H\&E staining was used for demonstrating presence of erythrocyte cells in the alveolar lumen. Slides were examined under $40 \mathrm{X}$ for the presence or absence of hemorrhage. At least $50 \%$ of each field was occupied by lung alveoli and fields consisting predominately of the lumen of large airways or vessels were rejected ${ }^{11}$. SPSS-21 was used to analyze the data. Quantitative variables were expressed as mean \pm standard deviation and the significant difference was determined using one way analysis of variance (ANOVA) followed by post Hoc Tukey test. Qualitative variables were expressed in frequency and percentage and compared by Pearson chi-square test. $p$-value of $\leq 0.05$ was considered significant.

\section{RESULTS}

Lung organ weight was measured using analytical balance The mean value of lung weight $(n=10)$ was highest in experimental group 2 i.e. $1.0750 \pm 0.17$ gm followed by experimental group 4 i.e. $1.0570 \pm 0.20$ gm. Lung weight in control group 1 and experimental group 3 were $0.7960 \pm 0.12$ and $0.9960 \pm 0.05$ gm respectively. To assess lung injury, RTBWI was calculated. Mean of RTBWI in control group 1 was $2.0179 \pm 0.16$, it was highest in experimental groups $2(2.6010 \pm 0.30)$ followed by group 4 i.e. $2.4170 \pm 0.52$ (fig-2).

Gross examination of lungs was carried out to assess the presence or absence of hemorrhagic foci (fig- 
1). On gross examination of lungs, control group A and

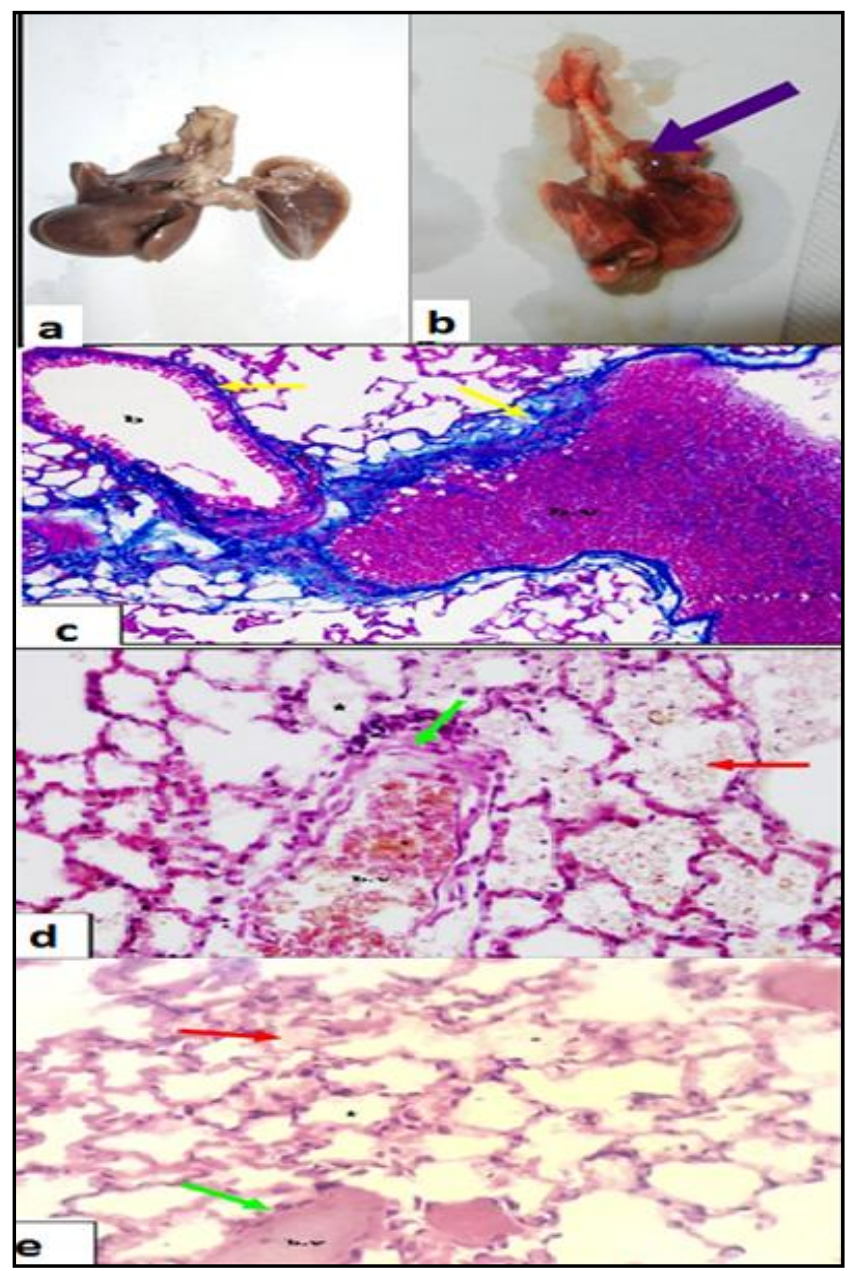

Figure-1(a-e): Photomicrograph (a \&b) demonstrating lungs of animal group $1 \& 2$ displaying normal and lungs showing haemorrhagic areas (purple arrow) respectively (c) experimental group 2 showing interstitial fibrosis. Collagen fibres (yellow arrow): 40X, Masson Trichrome. $N$ (d) Lungs of experimental Group 2 showing extensive extravasation of RBCs and thickened congested vessels, (e) experimental Group 4 showing few RBCs and normal blood vessels. RBCs (red arrows), blood vessel walls (green arrow), alveoli $\left({ }^{*}\right)$, blood vessels (b.v): 40X, H\&E.

experimental group $\mathrm{C}$ did not show any haemorrhagic foci. In all $10(100 \%)$ lungs of group B, dark haemorrhagic foci were observed (fig- $1 \mathrm{~b} \&$ fig- 3 ). Haemorrhagic foci were seen in only $5(50 \%)$ of lung specimens of group D (fig-3). Microscopic study was carried out and observations were made by examining H\&E stained slides under the light microscope at 40X magnification (fig-1) and extravasation of RBCs was noted inside the alveolar lumen and marked as present or absent. Coincidental with gross findings, all $10(100 \%)$ specimens of control group 1 and experimental group 3, did not show haemorrhage in three views of any slide. In expe- rimental group 2,100\% of specimens showed alveolar haemorrhages (fig-1d \& fig-3). In experimental group $4,5(50 \%)$ had A.H and rest of the $5(50 \%)$ did not show alveolar haemorrhages (fig-1e \& fig-3).

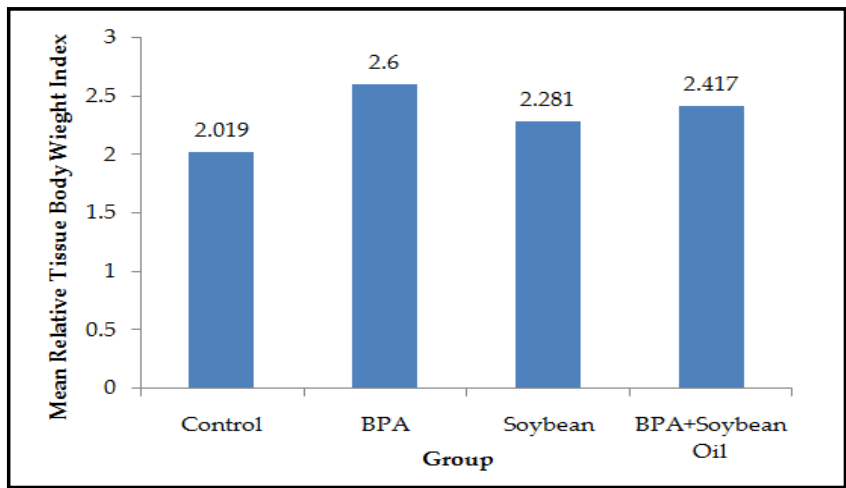

Figure-2: Comparison of mean relative tissue body weight index among the control group 1 and experimental groups 2,3 and 4 .

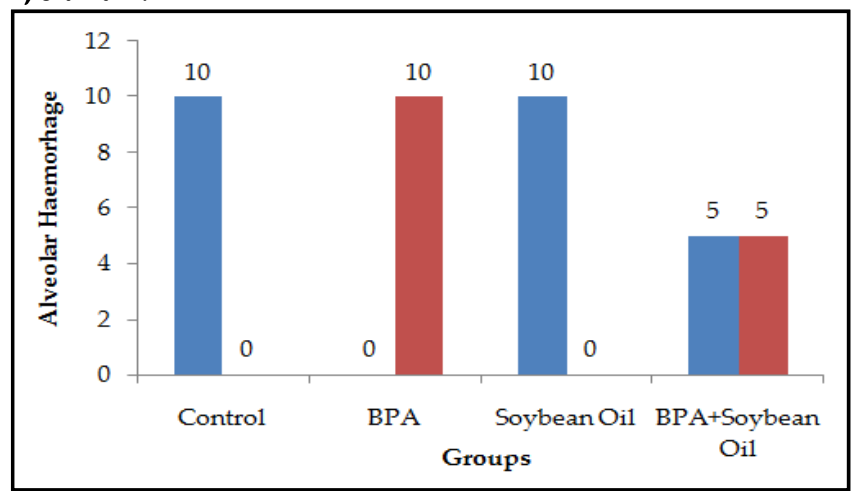

Figure-3: Alveolar haemorrhage among the control Group 1 and experimental groups 2, 3 and 4 .

\section{DISCUSSION}

The prevalent daily high utilisation of BPAcontaining products is of grave concern because of its adverse effects on different vital organs. Accordingly, this study was designed to study the effect of BPA on mice lungs along with administration of soybean oil that can have protective effect.

In the current study, eight of animal lungs in experimental group 2 documented increase in organ weight which are consistent with previous findings as a result of increased collagen deposition in interstitial connective tissue, diffuse inflammatory cellular infiltration, mild pulmonary edema and fatty infiltration due to increased expression of lipogenic genes ${ }^{12}$. Significant difference between lung weight of control group 1 was found with experimental groups $2(p$-value= $0.001), 3(p$-value $=0.028)$ and $4(p$-value $=0.003)$. This was in contrast to findings by Hassan et al in Riyadh, 
Saudi Arabia which stated that different doses of BPA have no effect on the absolute organ weight when compared to control groups ${ }^{13}$. Increase in lung weight in experimental group 3 was relevant to the body weight gain in that group as documented in another study carried out by Deol et al in California, United States of America ${ }^{14}$.

Absolute animal organ weight is not considered an ideal parameter for the estimation of experimental organ weight alterations, especially if co existent with differences in total body weight between the animal groups. In these scenarios, organ/body weight ratio or organ/brain weight ratio precisely identifies target organ toxicity ${ }^{10}$. In this study, RTBWI of experimental group 2 was found to be highest followed by experimental group 4 as is evident by the graph in fig- 2 . The $p$-value for RTBWI was highly significant when compared between control and experimental group as can be seen in table-II. compared to groups 1 and 3 ( $p$-value $=0.000$ ). Our current experiment proposes oxidative stress as an adverse effect of BPA by increase in immunoreaction for i-NOS and release of reactive oxygen species (ROS) ${ }^{15}$. Thus increasing vascular permeability and causing direct injury to vascular endothelium. Abedelhaffez, in Egypt proposed that Oxidative stress resulting from formation of ROS and quinine radicals cause pathological conditions including asthma, acute respiratory distress syndrome, chronic obstructive pulmonary disease (COPD) and idiopathic pulmonary fibrosis.

In the current study, co administration of soybean oil along with BPA significantly ameliorated BPAinduced variations. Soybean oil impairs inflammatory processes and provides ameliorative effects in terms of reduced inflammatory infiltrates, fibrosis and decreased thickness of alveolar septa along with diminished or absent intralveolar haemorrhages.

Similar findings were shown by Hillman et al,

Table-I: Intergroup comparison of $p$-value of lung weight, relative tissue body weight index (RTBWI) between control group 1 and experimental groups $2,3,4$.

\begin{tabular}{|c|c|c|c|c|c|c|c|c|c|c|}
\hline & \multicolumn{2}{|c|}{ Group 1 vs. 2} & \multicolumn{2}{|c|}{ Group 1 vs. 3} & \multicolumn{2}{|c|}{ Group 1 vs. 4} & Group 2 vs. 3 & Group 2 vs. 4 & Group 3 vs. 4 \\
\hline Weight of lungs & $\mathrm{gm})$ & \multicolumn{2}{|c|}{0.001} & \multicolumn{2}{|c|}{0.028} & \multicolumn{2}{|c|}{0.003} & 0.656 & 0.993 & 0.807 \\
\hline $\begin{array}{l}\text { Relative Body T } \\
\text { Weight Index (I }\end{array}$ & $\begin{array}{l}\text { sue } \\
\text { BWI) }\end{array}$ & \multicolumn{2}{|c|}{0.001} & \multicolumn{2}{|c|}{0.265} & \multicolumn{2}{|l|}{0.038} & 0.131 & 0.573 & 0.778 \\
\hline \multicolumn{11}{|c|}{ Table-II: Comparison of $p$-values of alveolar haemorrhage control group 1 and experimental groups 2,3 and 4.} \\
\hline & \multicolumn{2}{|c|}{ Group 1 vs. 2} & \multicolumn{2}{|c|}{ Group 1 vs. 3} & \multicolumn{2}{|c|}{ Group 1 vs. 4} & \multicolumn{2}{|c|}{ Group 2 vs. 3} & Group 2 vs. 4 & Group 3 vs. 4 \\
\hline $\begin{array}{l}\text { Alveolar } \\
\text { haemorrhages }\end{array}$ & \multicolumn{2}{|c|}{$<0.01$} & \multicolumn{2}{|c|}{$\begin{array}{c}\text { Not } \\
\text { computable }\end{array}$} & \multicolumn{2}{|r|}{0.033} & \multicolumn{2}{|r|}{$<0.01$} & 0.033 & 0.033 \\
\hline
\end{tabular}

Statistically significant difference was found on intergroup comparison of control group 1 with experimental group $2(p$-value $=0.001)$ and between control group 1 and experimental 4 ( $p$-value $=0.038)$. While when considering the RTBWI between experimental group 2 and group 4 the value was not significant that may indicate other factors which contribute to lung injury including inflammation, interstitial fibrosis and increased alveolar septal thickness and pulmonary oedema and these need to be investigated.

On gross examination, in experimental group 2, lungs were enlarged and edematous with dark hemorrhagic spots where as in control group 1 and experimental group 3, lungs appeared pink, soft and spongy in consistency. On intergroup comparison, statistical difference was present in lung weight of control group 1 with experimental groups 2,3 \& 4 .

Mice in experimental group 2 showed haemorrhagic foci and intra alveolar haemorrhages in $100 \%$ of specimens. These results were highly significant as
Detroit, Michigan USA that in non-small-cell lung cancer (NSCLC), soybean isoflavones given prior to radiation exposure and even subsequently had a protective effect against manifestation of adverse effects of radiation including inflamma-tion, pneumonitis and fibrosis ${ }^{16}$.

This outcome is supported by the fact that soyabean oil possesses anti-inflammatory ${ }^{17}$, and antioxidant properties ${ }^{18}$.

\section{ACKNOWLEDGMENT}

We were thankful to my supervisor and Head of department of Anatomy, AMC, Associate Prof. Brig. Dr. Shabnum Hamid and Prof. Brig. Khadija Qamar for their guidance and constant help throughout our work.

\section{CONCLUSION}

The study helps in identifying the protective effects of Soybean oil on lung injury due to Bisphenol A by causing decrease in fibrosis and inflammatory response. 


\section{CONFLICT OF INTEREST}

This study has no conflict of interest to be declared by any author.

\section{REFERENCES}

1. Gray JM, Rasanayagam S, Engel C, Rizzo J. State of the evidence 2017: an update on the connection between breast cancer and the environment. Environ Health 2017; 16(1): 94-100.

2. Huang FM, Chang YC, Lee SS, Yang ML, Kuan YH. Expression of pro-inflammatory cytokines and mediators induced by bisphenol A via ERK-NFKB and JAK1/2STAT3 pathways in macrophages. Environ Toxicol 2019; 34(4): 486-94.

3. Wang S, Yang Y, Luo D, Wu D, Liu H, Li M, et al. Lung inflammation induced by exposure to Bisphenol-A is associated with mTOR-mediated autophagy in adolescent mice. Chemosphere 2020; 248(2): 126035-38.

4. Karnam S, Ghosh R, Mondal M. Pathology of bisphenol A induced sub-acute toxicity in Albino rats. Indian J Vet Pathol 2016; 40(1): 47-50.

5. Wilson M, Wynn T. Pulmonary fibrosis: pathogenesis, etiology and regulation. Mucosal Immunol 2009; 2(2): 103-108.

6. Liu SH, Su CC, Lee KI, Chen YW. Effects of bisphenol a metabolite 4-Methyl-2,4-bis (4-hydroxyphenyl) pent-1-ene on lung function and type 2 pulmonary alveolar epithelial cell growth. Sci Rep 2016; 6(2): 39254-60.

7. Robinson L, Miller R. The Impact of Bisphenol A and Phthalates on Allergy, Asthma, and Immune Function: A Review of Latest Findings. Curr Environ Health Rep 2015; 2(4): 379-84.

8. Zhang Q, Tong X, Sui X, Wang Z, Qi B, Li Y, et al. Antioxidant activity and protective effects of Alcalase-hydrolyzed soybean hydrolysate in human intestinal epithelial Caco-2 cells. Food Res Int 2018; 111(2): 256-64.

9. Watanabe H, Sasatani M, Doi T, Masaki T, Satoh K, Yoshizumi M. Protective effects of Japanese soybean paste (miso) on stroke in stroke-prone spontaneously hypertensive rats (SHRSP). Am J Hypertens 2018; 31(1): 43-47.

10. Bailey SA, Zidell RH, Perry RW. Relationships between organ weight and body/brain weight in the rat: what is the best analytical endpoint?. Toxicol Pathol 2004; 32(4): 448-66.

11. Matute-Bello G, Downey G, Moore BB, Groshong SD, Matthay MA, Slutsky AS, et al. An official American thoracic society workshop report: features and measurements of experimental acute lung injury in animals. Am J Respir Cell Mol Biol 2011; 44(5): 725-38.

12. Marmugi A, Ducheix S, Lasserre F, Polizzi A, Paris A, Priymenko $\mathrm{N}$, et al. Low doses of bisphenol A induce gene expression related to lipid synthesis and trigger triglyceride accumulation in adult mouse liver. Hepatol 2012; 55(2): 395-407.

13. Hassan ZK, Elobeid MA, Virk P, Omer SA, ElAmin M, Daghestani $\mathrm{MH}$. Bisphenol A induces hepatotoxicity through oxidative stress in rat model. Oxid Med Cell Longev 2012; 2012(4): 1-5

14. Deol P, Evans JR, Dhahbi J, Chellappa K, Han DS, Spindler S, et al. Soybean oil is more obesogenic and diabetogenic than coconut oil and fructose in mouse: potential role for the liver. PLoS One 2015; 10(7): 1-4.

15. Sonavane M, Gassman NR. Bisphenol A co-exposure effects: a key factor in understanding BPA's complex mechanism and health outcomes. Crit Rev Toxicol 2019; 49(5): 371-86.

16. Hillman GG, Singh-Gupta V, Lonardo F, Hoogstra DJ, Abernathy LM, Yunker CK, et al. Radioprotection of lung tissue by soy isoflavones. J Thorac Oncol 2013; 8(11): 1356-64.

17. Navarro-Xavier RA, de Barros KV, de Andrade IS, Palomino Z, Casarini DE, Silveira VLF. Protective effect of soybean oil-or fish oil-rich diets on allergic airway inflammation. J Inflamm Res 2016; 9(2): 79-82.

18. Rodrigues H, Diniz Y, Faine L, Galhardi C, Burneiko R, Almeida $\mathrm{J}$, et al. Antioxidant effect of saponin: potential action of a soybean flavonoid on glucose tolerance and risk factors for atherosclerosis. Int J Food Sci Nutr 2005; 56(2): 79-85. 